\title{
The role of Sequence Type (ST) 131 in adult community-onset non-ESBL-producing Escherichia coli bacteraemia
}

\author{
Yi-Hui Wu ${ }^{1 \dagger}$, Ming-Fang Cheng ${ }^{2,3+}$, Chung-Hsu Lai ${ }^{1}$, Hsi-Hsun Lin ${ }^{1}$, Chih-Hsin Hung ${ }^{4^{*}}$ and Jiun-Ling Wang ${ }^{1 *}$
}

\begin{abstract}
Background: To compare the epidemiological and clinical features and outcome in clonal group O25b/ST131 and non-clonal group O25b/ST131 in adult patients with non-extended-spectrum B-lactamase (ESBL)-producing Escherichia coli (E. coli) bacteraemia.

Methods: We collected 371 consecutive isolates with community-onset non-ESBL producing E. coli bloodstream infection in 2010 in a 1200-bed hospital in Taiwan. Twenty adult patients with clonal group O25b/ST131 and 40 patients with non-clonal group O25b/ST131 were compared.

Result: Clonal group O25b/ST131 accounted for 5.9\% of total isolates. The underlying disease and healthcare-associated risk factors were similar in the case and control groups. Patients with the clonal group O25b/ST131 were less likely to have intra-abdominal infection (0\% vs. 22.5\%; $p<0.05)$ than patients from the control group. The Day 30 mortality rate was similar in the case and control groups (15\% vs. 12.5\%).

Conclusions: Clonal group O25b/ST131 was found in both multidrug-resistant and susceptible E. coli strains, causing community-onset bloodstream infection. Although O25b/ST131 does not lead to a higher mortality than other isolates, choosing an appropriate antimicrobials in the empirical therapy of community-onset $E$. coli bacteraemia has become more challenging.
\end{abstract}

Keywords: E. coli, Bacteraemia, Community onset, Fluoroquinolone resistant, ST (sequence type) 131, Mortality

\section{Background}

From 2000 to 2006, the Escherichia coli clone O25:H4ST131, which produces CTX-M-15 extended-spectrum Blactamase (ESBL), was identified in three continents [1-3]. ST131's distinctive combination of resistance and virulence, as well as its widespread dissemination among the locals, may underlie its epidemiologic success. Later studies have showed that clone ST131 exists in non-ESBL producing fluoroquinolone-resistant E. coli isolates [4-8]. ST131 accounted for $25 \%$ to $78 \%$ of fluoroquinolone resistant E. coli infection in the surveillance form in Asia and the US [3-8]. In our previous studies, fluoroquinolone

\footnotetext{
* Correspondence: chhung@isu.edu.tw; jiunlingwang@gmail.com ${ }^{\dagger}$ Equal contributors

${ }^{4}$ Department of Chemical Engineering, Institute of Biotechnology and Chemical Engineering, I-Shou University, Kaohsiung, Taiwan

'Department of Internal Medicine, E-Da Hospital, College of Medicine, I-Shou University, Kaohsiung, Taiwan

Full list of author information is available at the end of the article
}

(29\%) and cefazolin (25\%) resistant uropathogens were common in adults with community-onset urinary tract infections (UTI) in a teaching hospital in southern Taiwan. [9] There were several risk factor to be independently associated with community onset UTI fluoroquinolone resistant pathogens (i.e. recent hospitalization, underlying old stroke, and diabetes mellitus) [9]. Although cephalosporin and fluoroquinolone are the recommended antimicrobials to use in community-onset complicated UTI or bloodstream infection, the emergence of clone ST131 with fluoroquinolone resistance in acute pyelonephritis poses a challenge in choosing the adequate antimicrobials. In our institution, we often used $3^{\text {rd }}$ generation cephalosporin such as ceftriaxone or fluoroquinolone such as levofloxacin or ciprofloxacin as empirical antibiotics for community-onset complicated UTI or gram negative bacillary bacteremia. 
Our previous studies compared the clinical features and outcome in ST131 vs. non-ST131 in ESBL-producing E. coli bacteremia [10]. We found that ST131 was associated with non-catheter related UTI (urinary tract infection) and that it is not associated with higher mortality [10]. According to our review of the literature, we did not find clinical data for clone ST131 in community-onset ESBLnegative $E$. coli bacteremia. To further understand the role of ST131 in community-onset non-ESBL-producing E. coli bacteremia, we collected non-duplicated consecutive $E$. coli bloodstream infection isolates in 2010 in a medical center in southern Taiwan. Our goals were to determine the percentage of ST131 in community-onset non-ESBL-producing E. coli bloodstream infection and to find out if patients with ST131 clone have clinical features or outcome different from patients with a non-ST131 clone.

\section{Method}

Study design, setting and participants

The study was done retrospectively and included patients older than 16 years old with at least one positive blood culture of ESBL-negative $E$. coli who were admitted to the E-Da Hospital during a one-year period as Figure 1 (from
Jan 1, 2010 to Dec 31, 2010). E-Da Hospital is a 1200-bed major teaching hospital in southern Taiwan that provides both primary and tertiary medical care. Only strains from the first bacteremic episode were included in the analysis. Blood cultures were processed using the automated blood culture system. (BD Phoenix ${ }^{\mathrm{Tm}}$ Automated Microbiology System)

In the present study, non-susceptibility arbitrarily refers to the category of intermediate and resistant results, obtained using the MIC (minimal inhibitory concentration) value in accordance with the criteria from the Clinical and Laboratory Standards Institute, M100-S21.

\section{Variables, collection of data and definitions}

All patients were evaluated using a standard case recording form. Each clinical course of infection was evaluated and recorded according to the information supplied by primary care physicians and medical records reviewing retrospectively. The diagnosis of infection focus of bacteremia was based on clinical, bacteriological, and radiological investigations.

The following items were recorded for each patient: age; sex; underlying illness; infection syndrome type;

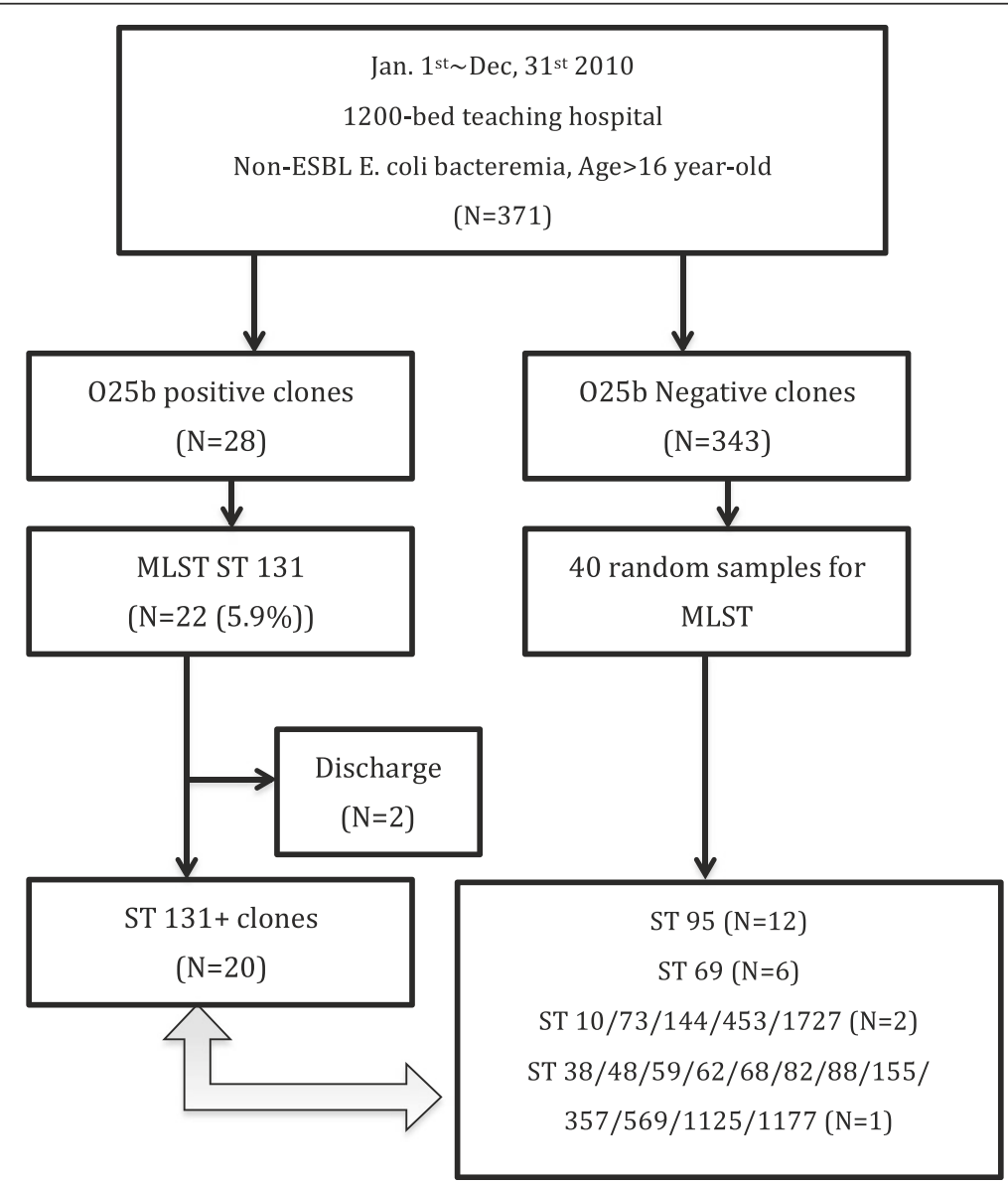

Figure 1 Algorism of methods and cases analyses. 
history of hospitalization within the previous three months; antimicrobials exposure within the previous three months, existence of a urinary catheter; initial empirical antimicrobial agents; and outcome. If an in vitro active antimicrobial agent was not administered before the final result of blood culture, the procedure was considered a discordant empirical therapy.

This study was supported by the E-Da Hospital Research Committee and approved by the institutional review board of E-Da Hospital. (EDAH IRB No./Protocol No: EMRP102-048). Because the study was done via medical chart reviewing retrospectively and the pathogens were collected from the stock of the infectious isolates from the laboratory. The IRB waived informed consent requirements due to the research involves no more than minimal risk to the subjects and the waiver will not affect adversely the rights and welfare of the patients.

\section{Microbiological studies}

O25 positve E. coli isolates were determined by the PCR method described by Clermont et al [2]. All O25b positive $E$. coli isolates were characterized by multilocus sequence typing (MLST) using the standard seven housekeeping genes (adk, fumC, gyrB, icd, mdh, purA, and recA), according to the protocol and primers specified by the $E$. coli MLST web site (http://mlst.warwick.ac.uk/mlst/ dbs/Ecoli) [2,11] MLST was also performed on a randomly selected subset of $40 \mathrm{O} 25 \mathrm{~b}$ negative E-coli isolates. Clonal relationships were established by pulsed-field gel electrophoresis (PFGE) of XbaI-digested genomic DNA. To compare the band patterns of aggregated data, the GelCompar software package (version 6.0; Applied Maths, Bionumerics) was used. Strains showing $80 \%$ or greater banding pattern similarity were considered to have similar or identical electrokaryotypes.

\section{Statistical analysis}

Descriptive statistics, including means, standard deviations, and ranges, were used to analyze continuous variables, whereas percentages and confidence intervals were used to analyze categorical variables. Independent-t test was used for continuous variables. A Chi-square test or Fisher's exact test was used for categorical variables. The variables in bivariate analyses with a $p$ value $<0.1$ were included in a multivariate analysis, which was performed using a logistic regression model to identify factors that independently and significantly affected the outcome. A $p$ value $<0.05$ was considered to be statistically significant. All statistical analyses were done using SPSS 15 for Windows, Chicago, IL, USA.

\section{Result}

Of the 371 non-duplicated consecutive E. coli bloodstream infection isolates, $43.9 \%, 30.7 \%, 15.4 \%, 15.4 \%, 13.2 \%$, and
4.6\% were resistant to trimethoprim/sulfamethoxazole (TMP/SMZ), amoxicillin/clavunate, cefazolin, gentamicin, ciprofloxacin, and cefotaxime, respectively (data not shown). The antibiotic resistance of all 371 isolates of clonal group O25b/ST131 and non clonal group O25b/ST131 were shown in the Additional file 1: Table S1. Clonal group 131 were more likely to have TMP/SMZ, ciprofloxacin, cefmetazole, and gentimicin resistance $(\mathrm{P}<0.05)$. Clonal group $\mathrm{O} 25 \mathrm{~b} / \mathrm{ST} 131(\mathrm{n}=22)$ accounted for $5.9 \%$ of the total isolates. Two cases of O25-ST131 did not have a complete history in our hospital and were excluded from further clinical analysis.

We collected clinical data from the remaining 20 cases of clonal group O25b/ST131 and 40 randomly selected cases of non-O25b/ST131 genotypes, which were used as controls. The most predominant ST types in the 40 non-O25b/ST131 isolates included ST95 $(\mathrm{n}=12)$ and ST $69(\mathrm{n}=6)$. The underlying co-morbidity and healthcareassociated risk factors (such as hospitalization and antimicrobials use) were similar in the case and control groups, as shown in Table 1. Patients with clonal group $\mathrm{O} 25 \mathrm{~b} / \mathrm{ST} 131$ were less likely to have intra-abdominal infection ( $0 \%$ vs. $22.5 \%$; $p<0.05)$ than patients with nonO25b/ST131 clones. Nine cases (15\%) received discordant antimicrobials before susceptibility data were available included levofloxacin $(n=4)$, cephazolin $(n=2)$, ceftriaxone $(\mathrm{n}=1)$, cefuroxime $(\mathrm{n}=1)$ and amoxicillin/clavunate $(n=1)$. There were $20 \%$ patients in clonal group O25b/ ST131 and $12.5 \%$ in non-clonal group O25b/ST131 that received discordant antimicrobials before susceptibility data were available $(p=0.464)$.

The Day 30 mortality rate was similar in the clonal group O25b/ST131 and non-clonal group O25b/ST131 $(12.5 \%$ vs. $15 \%, p=1.00)$, but patients infected with the clonal group $\mathrm{O} 25 \mathrm{~b} / \mathrm{ST} 131$ were more likely to have ESBL E. coli infection in the following two years than patients infected with the non-ST131 group (20\% vs. $2.5 \% p<0.05)$ (data not shown). However, we don't have the following ESBL E. coli isolates for further pulsotype study. The univariate risk factors for Day 30 mortality included underlying chronic renal failure (OR: 5.6; 95\% CI: 3.0-31.0; $p=$ 0.046) and solid organ cancer (OR: 7.0; 95\% CI: 1.4 -34.2; $p=0.016)$. The only independent risk factor of Day 30 mortality in multivariate analysis (logistic regression, backward Wald) was chronic renal failure (OR: 16.4; 95\% CI: $1.3-212.5 ; p=0.032$ ) and a primary urinary tract source of infection was associated with lower day 30 mortality (OR: 0.042; 95\% CI: 0.002-0.76; $p=0.032$ ) (Table 2). Clonal group O25b/ST131 was not related to Day 30 mortality in neither univariate nor multivariate analysis. The resistance percentage of these 60 isolates was shown on Table 3. The PFGE result is shown in Figure 2. There were several pulsotypes in clonal group O25b/ST131, and one major pulsotype included five isolates. 
Table 1 Clinical features and epidemiology data in cases with clonal group 025b/ST131 and control group

\begin{tabular}{|c|c|c|c|}
\hline \multirow[t]{2}{*}{ Parameter } & \multirow{2}{*}{$\begin{array}{l}\text { Non clonal group } \\
\text { O25b/ST131 } \\
\mathrm{N}=40\end{array}$} & $\begin{array}{l}\text { Clonal group } \\
\text { O25b/ST131 }\end{array}$ & \multirow[t]{2}{*}{$P$ Value } \\
\hline & & $N=20$ & \\
\hline Male sex & $10(25)$ & $8(40)$ & 0.232 \\
\hline Age & $67.0+-13.9$ & $66.0+/-17.7$ & 0.807 \\
\hline \multicolumn{4}{|l|}{ Underlying disease } \\
\hline Diabetes mellitus & $16(40.0)$ & $6(30)$ & 0.449 \\
\hline Hepatobiliary disease & $16(40.0)$ & $8(40)$ & 1.000 \\
\hline $\begin{array}{l}\text { Renal structure } \\
\text { abnormality }\end{array}$ & $3(7.5)$ & $4(20)$ & 0.208 \\
\hline Chronic renal disease & $4(10)$ & $4(20)$ & 0.422 \\
\hline Liver cirrhosis & $8(20)$ & $2(10)$ & 0.471 \\
\hline Malignancy & $8(20)$ & $7(35)$ & 0.206 \\
\hline Bedridden & $4(10)$ & $2(10)$ & 1.000 \\
\hline \multicolumn{4}{|l|}{$\begin{array}{l}\text { Healthcare-associated } \\
\text { risk factor }\end{array}$} \\
\hline $\begin{array}{l}\text { Hospitalization in } \\
\text { previous } 6 \text { months }\end{array}$ & $20(50)$ & $6(30)$ & 0.141 \\
\hline \multicolumn{4}{|l|}{$\begin{array}{l}\text { Antimicrobials exposure } \\
\text { in } 3 \text { months }\end{array}$} \\
\hline B-lactam antimicrobials & $17(42.5)$ & $6(30)$ & 0.102 \\
\hline Fluoroquinolone & $0(0)$ & $2(10)$ & \\
\hline Recent operation & $5(12.5)$ & $1(5)$ & 0.653 \\
\hline With Foley catheter & $5(12.5)$ & $0(0)$ & 0.159 \\
\hline \multicolumn{4}{|l|}{ Infection syndrome } \\
\hline Urinary tract infection & $22(55.0)$ & $12(60)$ & 0.713 \\
\hline Intra-abdominal infection & $9(22.5)$ & $0(0)$ & 0.023 \\
\hline \multicolumn{4}{|l|}{ Outcome } \\
\hline Discordant antimicrobials & $5(12.5)$ & $4(20)$ & 0.464 \\
\hline Shock & 6(15) & $4(20)$ & 0.718 \\
\hline Length of stay $>2$ weeks & $11(27.5)$ & $6(30)$ & 0.839 \\
\hline Day 30 mortality & $5(12.5)$ & $3(15)$ & 1.000 \\
\hline
\end{tabular}

\section{Discussion}

We have shown that O25-ST131 is also the important clone in non-ESBL-producing E. coli bacteremia. Clonal group O25b/ST131 accounted for 5.9\% in total isolates and had a higher proportion in gentamicin, TMP/SMZ and fluoroquinolone resistant isolates. Clonal group

Table 2 Univariate and multivariate analysis in risk factor of Day 30 mortality

\begin{tabular}{lll}
\hline Parameter & $\begin{array}{l}\text { Univariate } \\
\text { Crude OR (95\% CI) }\end{array}$ & $\begin{array}{l}\text { Multivariate } \\
\text { Adjusted OR (95\% CI) }\end{array}$ \\
\hline Chronic renal disease & $5.6(3.0-31.0)$ & $16.4(1.3-212.5)$ \\
Urinary tract infection & $0.08(0.09-0.72)$ & $0.042(.002-0.76)$ \\
Malignancy & $7.0(1.4-34.2)$ & Not significant \\
Clonal group O25b/ST131 & $1.002(0.99-1.013)$ & Not significant \\
\hline
\end{tabular}

Table 3 Percentage of antibiotic resistance in ST 131 clones and nonST131 clones ( $N=60)$

\begin{tabular}{llll}
\hline $\begin{array}{l}\text { Resistance percentage } \\
\text { (\%) }\end{array}$ & $\begin{array}{l}\text { O25b-ST131 } \\
\mathbf{N = 2 0}\end{array}$ & $\begin{array}{l}\text { Non O25b-ST131 } \\
\mathbf{N = 4 0}\end{array}$ & P value \\
\hline Amox/Clavu & $6(30)$ & $12(30)$ & \\
TMP/SMZ & $14(70)$ & $20(50)$ & 0.788 \\
Ciprofloxacin & $7(35)$ & $4(10)$ & 0.141 \\
Cefazolin & $6(30)$ & $5(12.5)$ & 0.018 \\
Cefmetazole & $4(20)$ & $1(2.5)$ & 0.099 \\
Cefotaxime & $2(10)$ & $0(0)$ & 0.038 \\
Piperacillin & $16(80)$ & $26(65)$ & 0.107 \\
Gentamicin & $11(55)$ & $6(15)$ & 0.232 \\
\hline
\end{tabular}

Note: Amox/Clavu denotes as Amoxicillin/Clavunic acid, TMP/SMZ denotes as Trimethoprim/sulfamethoxazole.

No resistance of cefepime, imipenem, amikacin found in these two groups.

O25b/ST131 existed in both multidrug-resistant and susceptible E. coli strains causing community-onset bloodstream infection. Most cases with clonal group O25b/ ST131 did not have apparent healthcare associated risk factors. This finding answers a previous suggestion that the emerging of clonal group O25b/ST131 may be explained by the enhanced ability to cause extraintestinal infection rather than selection by antimicrobial agents used in hospitals. Current study excluding ESBL positive isolates showed approximately one fourth of fluoroquinolone resistant E. coli bacteremia was O25-ST131. Similar to a previous study, although more present in multidrugresistant isolates, ST131 was found in both antimicrobials susceptible and resistant blood isolates [11,12]. In a bacteremia study in San Francisco, ST131 accounted for approximately $20 \%$ of all drug-resistant and susceptible isolates [11]. Uchida et al. investigated 219 fluoroquinoloneresistant $E$. coli strains in Asian and found that 32\% strains were from the serogroup O25 [7]. In Korea, 25\% of community-onset urine E. coli isolates were ST131 from November 2006 to August 2007 [8].

Similar to a previous study, O25-ST131 was less likely to be associated with intra-abdominal infection and more likely to be related to UTI [5]. In a study from Spain, some healthcare associated risk factors such as diabetes, bedridden status, and antimicrobials exposure were independent risk factors for clone ST131 [13]. A trend for fluoroquinolone exposure in clonal group ST131/O25b was observed in our study but did not reach statistical significance. However, the other correlations were not found in our series, which is possibly explained by the fact that we included only community-onset bacteremia, and patients with some healthcare-associated risk factors may have been excluded. Similar to the study in Spain, mortality was similar between the ST131 and non-ST131 groups [13]. Our study is also in agreement with a previous murine sepsis model in which neither ST131 status nor fluoroquinolone resistance 


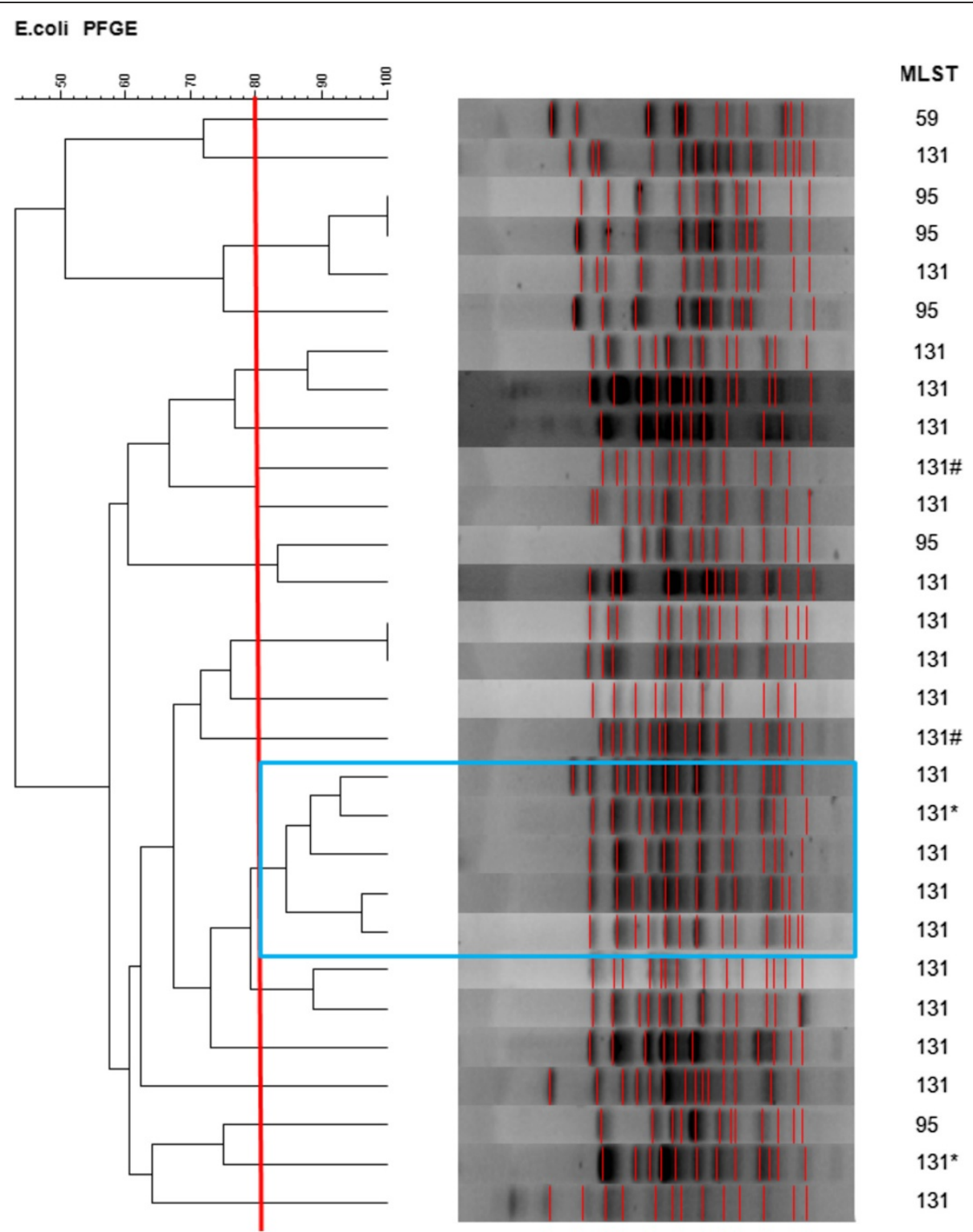

Figure 2 Pulsed-field gel electrophoresis (PFGE) of Xbal-digested genomic DNA of 23 clonal group 025b/ST131 isolates and five isolates with ST95 and one isolate with ST59. The major pulsotype was marked. Note: 1. One case with repeated bacteremia (two isolates \#) in the same year. 2. Two isolates* were excluded from the clinical analysis due to incomplete clinical data in the medical chart.

correlated with mortality [14]. More cases of clone ST131 had a higher chance of ESBL E. coli infection in the following two years than cases of non-ST131. It remains to be clarified if this phenomenon is related to the colonization and transmission features of ST 131. Nevertheless, a study from France showed that ST131 had high intestine colonization and urinary tract infection abilities, and according to a recent report, ST131 clone was associated with recurrent or persistent urinary tract infection $[15,16]$.

In our study, despite the high percentage use of discordant antimicrobials in both the ST131 and nonST131 groups, the mortality was not changed much. Taking into account the predominance of ST131 in
E. coli bacteremia infection, the selection of adequate empirical antimicrobials becomes difficult. In Asia, the susceptibility rate to fluoroquinolones in $E$. coli was approximately $70 \%$ in the urinary isolates from the SMART study [17]. In community-acquired bacteremic acute pyelonephritis, discordant empirical therapy was associated with a worse early clinical response and longer hospital stay than in concordant therapy [18-20]. However, choosing broadspectrum antimicrobials such as carbapenem in this type of community-onset infection raises the concern of antimicrobials selection pressure.

This study is limited by the following factors. First, this is a single center study in Taiwan that included a moderate 
number of case numbers, and we do not know if the result can be generalized to other parts of the world. Second, we did not collect isolates from colonization or infection after the bacteremia episode. We ignore if the ESBL isolates cultured after infection have the same genotype as the isolates collected during the non-ESBL producing $E$. coli bacteremia. Third, we used the O25b PCR as a screen and confirm by MLST to identify the clonal group O25b/ ST131, some of isolates of ST131 but O25b negative may be missed in this screening method. The clinical significance of clone O25b negative ST131 was not known.

\section{Conclusion}

Our preliminary study shows that clone O25b-ST131 isolates emerged as an important cause of non-ESBLproducing E. coli bacteremia in Taiwan. Clonal group $\mathrm{O} 25 \mathrm{~b} / \mathrm{ST} 131$ existed in both multidrug-resistant and susceptible E. coli strains causing community-onset bloodstream infection. Although O25b/ST131 does not lead to a higher mortality than other isolates, choosing an appropriate antimicrobials in the empirical therapy of communityonset $E$. coli bacteremia has become more challenging due to the appearance of the ST131 clone.

\section{Additional file}

Additional file 1: Table S1. Percentage of antibiotic resistance in ST 131 clones and non ST131 clones in all isolates $(\mathrm{N}=371)$.

\section{Competing interests}

The authors declare that they have no competing interests.

\section{Authors' contributions}

YHW, JLW, CHH carried out the molecular genetic studies and drafted the manuscript. $\mathrm{CHL}$ and $\mathrm{HHL}$ participated in the design of the study and collected the bacteria isolates. MFC participated in its design and coordination and helped to draft the manuscript. All authors read and approved the final manuscript.

\section{Acknowledgements}

This work was supported by research grants (EDAHP103057) from E-DA hospital. The funders had no role in study design, data collection and analysis, decision to publish, or preparation of the manuscript.

\section{Author details \\ 'Department of Internal Medicine, E-Da Hospital, College of Medicine, I-Shou University, Kaohsiung, Taiwan. ${ }^{2}$ Department of Pediatrics, Kaohsiung Veterans General Hospital, Kaohsiung, Taiwan. ${ }^{3}$ School of Medicine, National Yang-Ming University, Taipei, Taiwan. ${ }^{4}$ Department of Chemical Engineering, Institute of Biotechnology and Chemical Engineering, I-Shou University, Kaohsiung, Taiwan.}

Received: 31 May 2014 Accepted: 22 October 2014

Published online: 07 November 2014

\section{References}

1. Nicolas-Chanoine MH, Blanco J, Leflon-Guibout V, Demarty R, Alonso MP, Caniça MM, Park YJ, Lavigne JP, Pitout J, Johnson JR: Intercontinental emergence of Escherichia coli clone O25:H4-ST131 producing CTX-M-15. J Antimicrob Chemother 2008, 61:273-281.

2. Clermont O, Dhanji H, Upton M, Gibreel T, Fox A, Boyd D, Mulvey MR, Nordmann P, Ruppé E, Sarthou JL, Frank T, Vimont S, Arlet G, Branger C,
Woodford N, Denamur E: Rapid detection of the O25b-ST131 clone of Escherichia coli encompassing the CTX-M-15-producing strains. J Antimicrob Chemother 2009, 64:274-277.

3. Peirano G, Pitout JD: Molecular epidemiology of Escherichia coli producing CTX-M beta-lactamases: the worldwide emergence of clone ST131 O25:H4. Int J Antimicrob Agents 2010, 35:316-321.

4. Johnson JR, Nicolas-Chanoine MH, DebRoy C, Castanheira M, Robicsek A, Hansen G, Weissman S, Urban C, Platell J, Trott D, Zhanel G, Clabots C, Johnston BD, Kuskowski MA, MASTER Investigators: Comparison of Escherichia coli ST131 pulsotypes, by epidemiologic traits, 1967-2009. Emerg Infect Dis 2012, 18:598-607.

5. Johnson JR, Johnston B, Clabots C, Kuskowski MA, Castanheira M: Escherichia coli sequence type ST131 as the major cause of serious multidrug-resistant E. coli infections in the United States. Clin Infect Dis 2010, 51:286-294.

6. Yokota S, Sato T, Okubo T, Ohkoshi Y, Okabayashi T, Kuwahara O, Tamura Y, Fujii N: Prevalence of fluoroquinolone-resistant Escherichia coli O25:H4-ST131 (CTX-M-15-nonproducing) strains isolated in Japan. Chemotherapy 2012, 58:52-59.

7. Uchida Y, Mochimaru T, Morokuma Y, Kiyosuke M, Fujise M, Eto F, Eriguchi Y, Nagasaki Y, Shimono N, Kang D: Clonal spread in Eastern Asia of ciprofloxacin-resistant Escherichia coli serogroup $\mathrm{O} 25$ strains, and associated virulence factors. Int J Antimicrob Agents 2010, 35:444-450.

8. Lee MY, Choi HJ, Choi JY, Song M, Song Y, Kim SW, Chang HH, Jung SI, Kim YS, Ki HK, Son JS, Kwon KT, Heo ST, Yeom JS, Shin SY, Chung DR, Peck KR, Song JH, Ko KS: Dissemination of ST131 and ST393 community-onset, ciprofloxacin-resistant Escherichia coli clones causing urinary tract infections in Korea. J Infect 2010, 60:146-153.

9. Wu YH, Chen PL, Hung YP, Ko WC: Risk factors and clinical impact of levofloxacin or cefazolin nonsusceptibility or ESBL production among uropathogens in adults with community-onset urinary tract infections. J Microbiol Immunol Infect 2012, Oct 11(E-Pub).

10. Chung HC, Lai CH, Lin JN, Huang CK, Liang SH, Chen WF, Shih YC, Lin HH Wang JL: Bacteremia caused by extended-spectrum- $\beta$-lactamase-producing Escherichia coli sequence type ST131 and non-ST131 clones: comparison of demographic data, clinical features, and mortality. Antimicrob Agents Chemother 2012, 56:618-622.

11. Adams-Sapper S, Diep BA, Perdreau-Remington F, Riley LW: Clonal composition and community clustering of drug-susceptible and -resistant Escherichia coli isolates from bloodstream infections. Antimicrob Agents Chemother 2013, 57:490-497.

12. Horner C, Fawley W, Morris K, Parnell P, Denton M, Wilcox M: Escherichia coli bacteraemia: 2 years of prospective regional surveillance (2010-12). J Antimicrob Chemother 2014, 69:91-100.

13. López-Cerero L, Navarro MD, Bellido M, Martín-Peña A, Viñas L, Cisneros JM, Gómez-Langley SL, Sánchez-Monteseirín H, Morales I, Pascual A, RodríguezBaño J: Escherichia coli belonging to the worldwide emerging epidemic clonal group 025b/ST131: risk factors and clinical implications. J Antimicrob Chemother 2014, 69:809-814.

14. Johnson JR, Porter SB, Zhanel G, Kuskowski MA, Denamur E: Virulence of Escherichia coli clinical isolates in a murine sepsis model in relation to sequence type ST131 status, fluoroquinolone resistance, and virulence genotype. Infect Immun 2012, 80:1554-1562

15. Vimont S, Boyd A, Bleibtreu A, Bens M, Goujon JM, Garry L, Clermont O, Denamur E, Arlet G, Vandewalle A: The CTX-M-15-producing Escherichia coli clone O25b: H4-ST131 has high intestine colonization and urinary tract infection abilities. PLoS One 2012, 7:e46547.

16. Tchesnokova V, Billig M, Chattopadhyay S, Linardopoulou E, Aprikian P, Roberts PL, Skrivankova V, Johnston B, Gileva A, Igusheva I, Toland A, Riddell K, Rogers P, Qin X, Butler-Wu S, Cookson BT, Fang FC, Kahl B, Price LB, Weissman SJ, Limaye A, Scholes D, Johnson JR, Sokurenko EV: Predictive diagnostics for Escherichia coli infections based on the clonal association of antimicrobial resistance and clinical outcome. J Clin Microbio/ 2013, 51:2991-2999.

17. Lu PL, Liu YC, Toh HS, Lee YL, Liu YM, Ho CM, Huang CC, Liu CE, Ko WC, Wang $J$ H, Tang HJ, Yu KW, Chen YS, Chuang YC, Xu Y, Ni Y, Chen YH, Hsueh PR: Epidemiology and antimicrobial susceptibility profiles of Gram-negative bacteria causing urinary tract infections in the Asia-Pacific region: 2009-2010 resultsfrom the Study for Monitoring Antimicrobial Resistance Trends (SMART). Int JAntimicrob Agents 2012, 40(Suppl):S37-S43.

18. Lee SS, Kim Y, Chung DR: Impact of discordant empirical therapy on outcome of community-acquired bacteremic acute pyelonephritis. J Infect 2011, 62:159-164. 
19. Johnson L, Sabel A, Burman WJ, Everhart RM, Rome M, Mackenzie TD, Rozwadowski J, Mehler PS, Price CS: Emergence of fluoroquinolone resistance in outpatient urinary Escherichia coli isolates. Am J Med 2008, 121:876-884.

20. van der Starre WE, van Nieuwkoop C, Paltansing S, van't Wout JW, Groeneveld GH, Becker MJ, Koster T, Wattel-Louis GH, Delfos NM, Ablij HC, Leyten EM, Blom $J$ W, van Dissel JT: Risk factors for fluoroquinolone-resistant Escherichia coli in adults with community-onset febrile urinary tract infection. J Antimicrob Chemother 2011, 6:6650-6656.

doi:10.1186/s12879-014-0579-z

Cite this article as: Wu et al:: The role of Sequence Type (ST) 131 in adult community-onset non-ESBL-producing Escherichia coli bacteraemia. BMC Infectious Diseases 2014 14:579.

\section{Submit your next manuscript to BioMed Central and take full advantage of:}

- Convenient online submission

- Thorough peer review

- No space constraints or color figure charges

- Immediate publication on acceptance

- Inclusion in PubMed, CAS, Scopus and Google Scholar

- Research which is freely available for redistribution 